\title{
A novel simple technique to enlarge the aortic annulus by two valve sizes
}

\author{
Bo Yang, MD, PhD, Ann Arbor, Mich
}

\footnotetext{
From the Department of Cardiac Surgery, Michigan Medicine, Ann Arbor, Mich.

Dr Yang is supported by the National Heart, Lung, and Blood Institute of the National Institutes of Health K08HL130614, R01HL141891, and R01HL151776, Phil Jenkins and Darlene \& Stephen J. Szatmari Funds. Disclosures: The author reported no conflicts of interest.

The Journal policy requires editors and reviewers to disclose conflicts of interest and to decline handling or reviewing manuscripts for which they may have a conflict of interest. The editors and reviewers of this article have no conflicts of interest.

Received for publication Oct 23, 2020; accepted for publication Oct 27, 2020; available ahead of print Nov 5 , 2020.

Address for reprints: Bo Yang, MD, PhD, 5155 Frankel Cardiovascular Center, 1500 East Medical Center Dr, Ann Arbor, MI 48109 (E-mail: boya@med.umich.edu).

JTCVS Techniques 2021;5:13-6

2666-2507

Copyright (C) 2020 The Authors. Published by Elsevier Inc. on behalf of The American Association for Thoracic Surgery. This is an open access article under the CC BY-NC-ND license (http://creativecommons.org/licenses/bync-nd/4.0/).

https://doi.org/10.1016/j.xjtc.2020.10.038
}

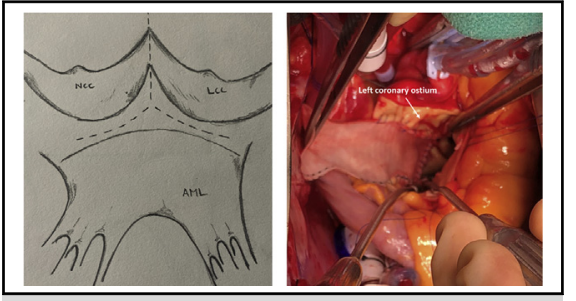

$Y$ incision at aortomitral curtain $(L)$ and rectangular patch to enlarge aortic root (R).

\section{CENTRAL MESSAGE \\ $Y$ incision at the aortomitral curtain and rectangular patch enlarged the aortic root by 2 sizes without violating the mitral valve.}

See Commentaries on pages 17 and 19.
Video clip is available online.

The current techniques of aortic root enlargement used by adult cardiac surgeons are the Nicks ${ }^{1}$ and Manouguian procedures. $^{2}$ The Nicks procedure generally increases aortic annulus by one valve size. ${ }^{1}$ The Manouguian requires incising the mitral valve (MV) anterior leaflet and left atrium (LA), with risk of mitral regurgitation. ${ }^{3}$ Reported herein is a new surgical technique to enlarge the aortic annulus by 2 valve sizes without violating the LA or MV.

\section{CASE REPORT}

A 62-year-old female patient with body height of $5^{\prime} 2^{\prime \prime}$ $(158 \mathrm{~cm})$ and surface area of $1.66 \mathrm{~m}^{2}$, status post combined pancreas-kidney transplant and second kidney transplant with significant renal dysfunction-presented with acute myocardial infarction, severe aortic stenosis, and a left ventricular ejection fraction of $35 \%$. She underwent urgent 4-vessel coronary artery bypass, aortic annular enlargement, and aortic valve replacement (AVR). The annulus size was measured at $19 \mathrm{~mm}$ after excision of the leaflets and annular debridement of calcium. After an aortic root enlargement procedure, a 23-mm Magna Ease (Edwards Lifesciences; Irvine, Calif) aortic bioprosthesis was implanted. Postoperative aortic valve mean gradient was $7 \mathrm{~mm} \mathrm{Hg}$, and aortic root diameter increased from 23 to $30 \mathrm{~mm}$ with normal coronary blood flow and no mitral regurgitation. This patient was discharged without complications, and provided informed consent for publication of the information included in this case report.

\section{Details of Aortic Root Enlargement (Video 1)}

A partial transverse aortotomy was made $1.5 \mathrm{~cm}$ above the sinotubular junction and stopped above the left-non commissure post. The stenotic aortic valve was excised

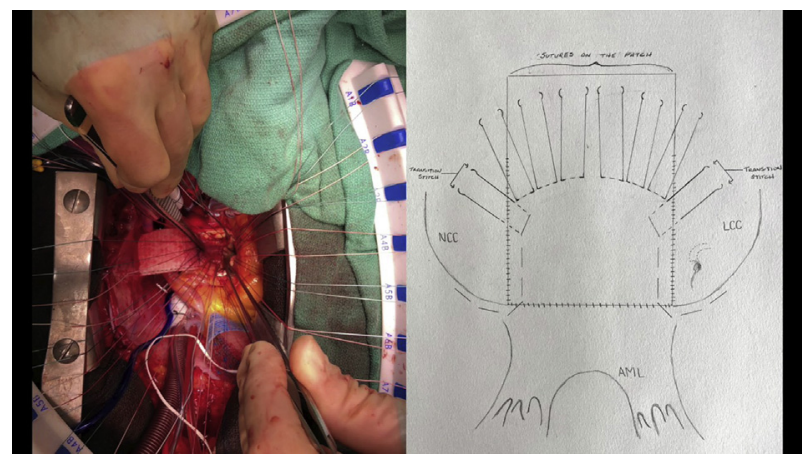

VIDEO 1. Discussion of the technique details, tips, and pitfalls of aortic annulus enlargement with a "Y" incision and rectangular patch with an additional case presentation, intraoperative video, photos, and illustrations Video available at: https://www.jtcvs.org/article/S2666-2507(20)30627-1/ fulltext. 

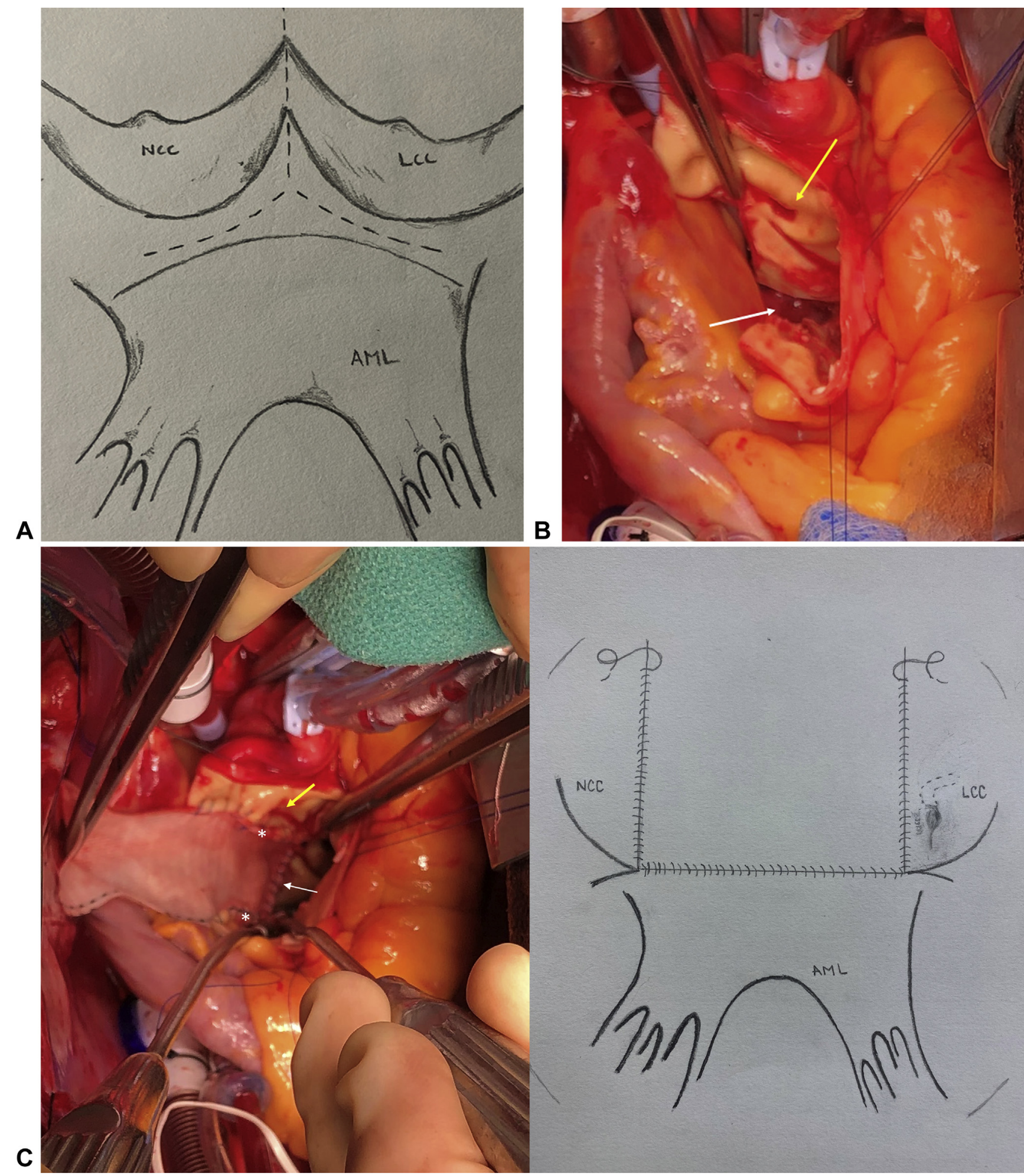

FIGURE 1. A, A "Y" incision through the left-non commissure post extended below the aortic annulus to the area between the nadirs of left and noncoronary cusps and mitral valve annulus for the aortic root enlargement. B, The aortic root was wide open after the Y incision (white arrow). Yellow arrow shows the left coronary ostium. C, In the left panel, a rectangular patch was sewn to the mitral annulus (white arrow) at the bottom and aortic annulus (white stars) on both sides, then to the incision of the transverse aortotomy. Yellow arrow shows left coronary ostium. In the right panel, an illustration of the suture lines of the anastomosis of the Dacron patch to the mitral and aortic annulus. 
and the annulus was debrided of calcium. The left-non commissure post was incised from the aortotomy into the aortomitral curtain close to the left atrial dome. The incision was extended in a "Y" fashion undermining the left and noncoronary annulus to their respective nadir (Figure 1, $A$ ). This maneuver widely opens the aortic root (Figure 1, $B$ ). A rectangular-shaped Hemashield Dacron patch (Boston Scientific Corp, Natick, Mass) was trimmed in width slightly greater than the distance between the 2 cusp nadirs. This patch was sewn to the mitral annulus from left to right fibrous trigone with running 4-0 PROLENE suture (Ethicon, Somerville, NJ). The suture line was transitioned to the undermined aortic annulus at the nadir of both left and noncoronary sinuses, sutured along the longitudinal length of the patch up to the level of the transverse aortotomy incision, and secured (Figures 1, $C$, and 2). The upsized valve sizer was placed in the enlarged root touching 3 nadirs of aortic annulus and the position of the sizer on the patch was marked to guide the placement of valve sutures (Figure 3, A). The non-pledgetted 2-0 ETHIBOND sutures (Ethicon) were placed along the native aortic annulus in a non-everting fashion and from outside in on the patch (Figure 3, B). The bioprosthesis was placed with one strut facing the left-right commissure post, one strut close to right-non commissure post, and one facing the patch (Figure 3,C). The sutures at nadirs of noncoronary and left coronary sinuses, which were the lowest point of aortic annulus, were tied first to seat the valve well and prevent paravalvular leak. A portion of the patch lay beneath the prosthetic valve thereby enlarging the root and left ventricular outflow tract (Figure 3,D). The aortotomy was then closed from the left to the right incorporating the patch (Figure 3, E).

\section{DISCUSSION}

A small aortic root can cause patient-prosthesis mismatch and exclude patients from transcatheter AVR. Our concept is similar to the Nicks and Manouguian procedures-to enlarge the fibrous portion of the root and subsequently the aortic annulus. The difference is our technique radically enlarges the fibrous portion of the aortic root by replacing the entire aortomitral curtain with a rectangular patch to accommodate an increase of 2 valve sizes from the initial assessment without violating the LA or MV. This enlargement technique originated from our experience of reconstructing the aortic root after the aortomitral curtain was completely destroyed by removing an old mechanical valve or endocarditis in small roots. In those cases, once the aortic annulus was incised, a large patch to repair the aortomitral curtain significantly enlarged the aortic annulus to allow a bigger prosthetic valve. Sewing the patch to the mitral and aortic annulus is more secure compared with the Nicks and Manouguian procedures, which sew the patch to the aortomitral curtain - a thin layer of fibrous tissue. We recommended a " $\mathrm{Y}$ " incision instead of a " $\mathrm{T}$ " incision to avoid accidental incising the LA or MV since sometimes it is difficult to differentiate the aortomitral curtain, mitral annulus, and MV. The "Y" incision could be easily extended below the nadirs of aortic annulus from aortomitral curtain without requiring dissecting out of aortic root but should not pass the fibrous tissue under nadirs of both left and noncoronary sinuses. The rectangular patch could push on the left coronary sinus with a potential of distortion of left coronary artery. However, the transverse sinus behind the rectangular patch allows the patch and left coronary sinus to expand posteriorly without significant distortion of left coronary artery. Because of this potential risk, we do not recommend
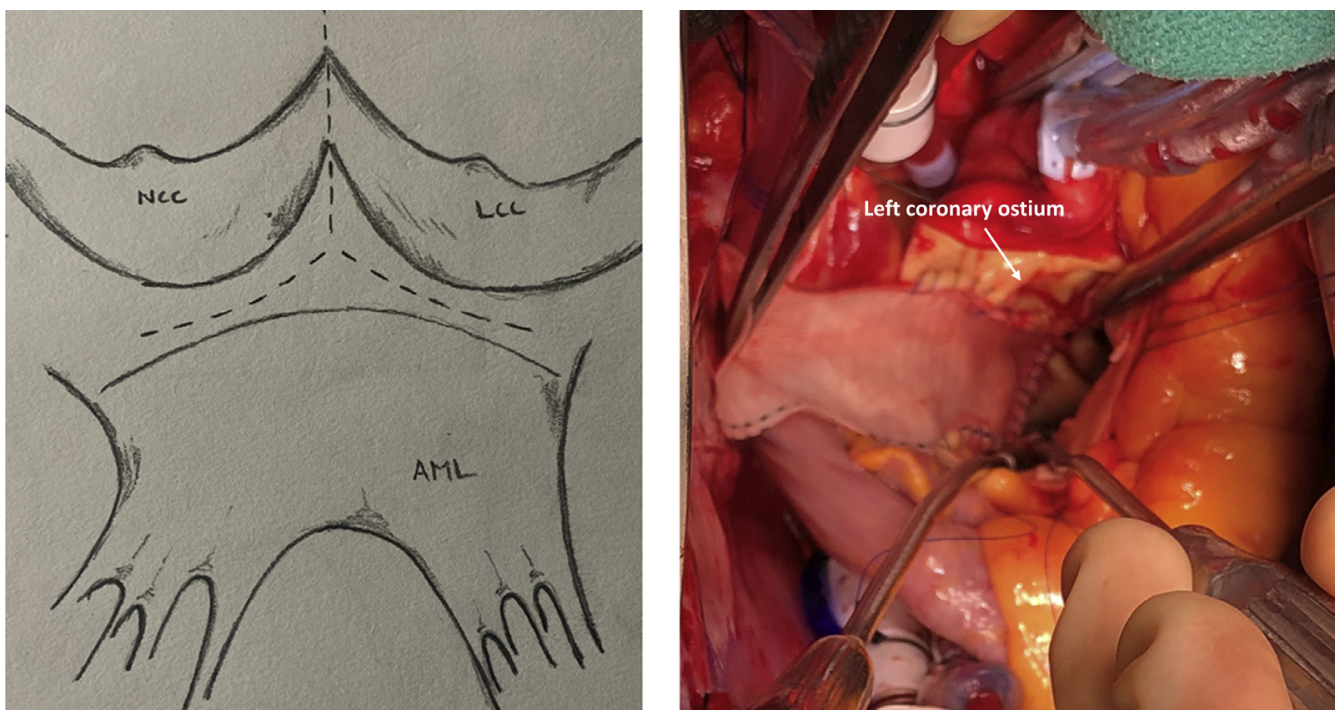

FIGURE 2. Y incision at aortomitral curtain (left) and rectangular patch to enlarge aortic root (right). 

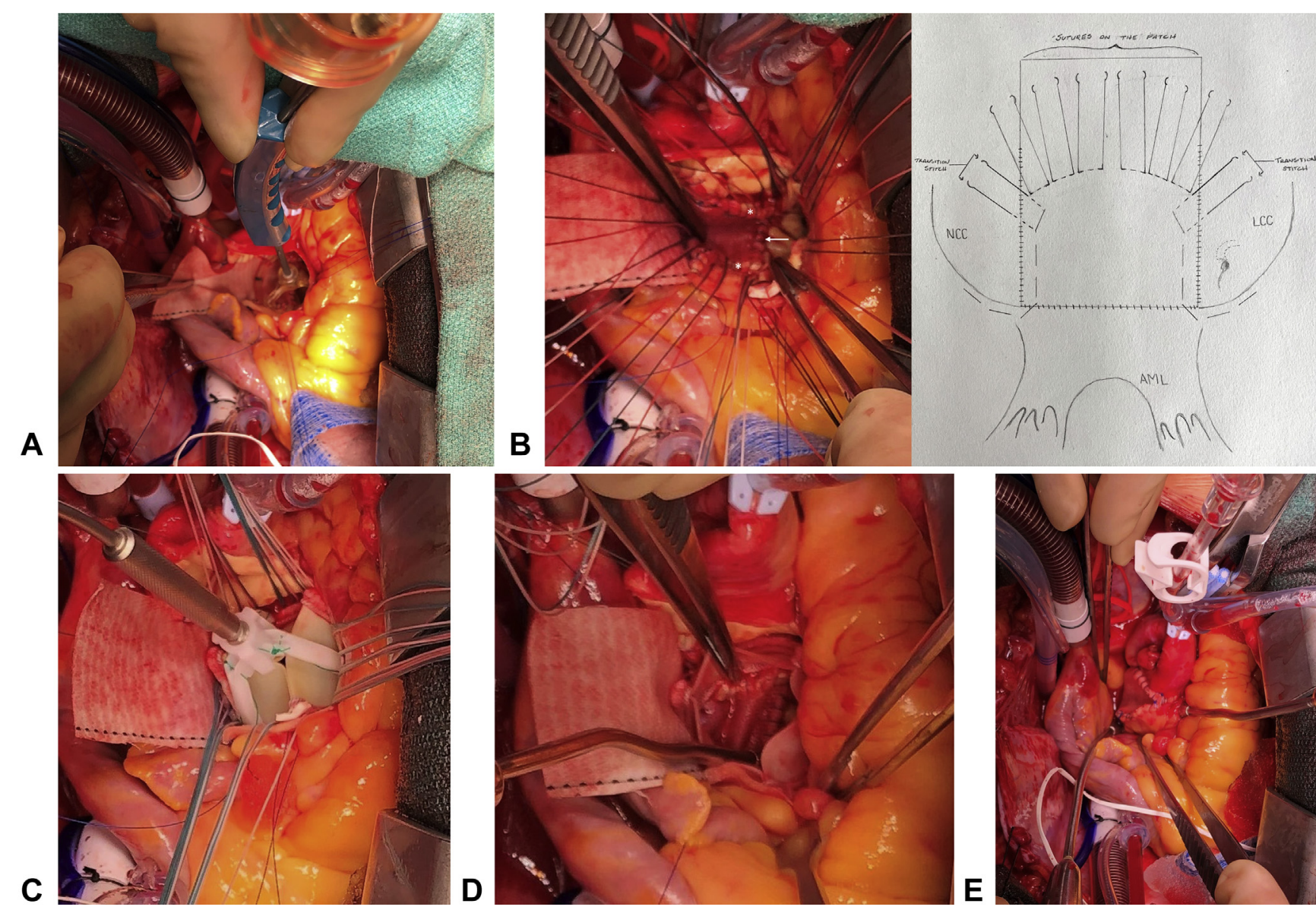

FIGURE 3. A, The valve position was marked on the Dacron patch to guide the placement of valve sutures with the 2-size larger sizer touching 3 nadirs of the aortic annulus of 3 aortic cusps. B, 2-0 ETHIBOND sutures were placed along the aortic annulus in a non-everting fashion and from outside in on the patch (left panel, white arrow: mitral annulus; white stars: aortic annulus sewn to the patch). The transitional stitches were placed with one arm of the suture through the aortic annulus (non-everting: from ventricle to aorta), and the other arm through the patch (one bite: inside-outside-inside) (right panel: illustration). C, The upsized bioprosthetic valve was seated right above the aortic annulus and the Dacron patch with one strut facing the left-right commissure post to position the coronary ostia above the nadir of left and right coronary cusps of the bioprosthesis. D, The left ventricular outflow tract was wide open underneath the upsized bioprosthesis (view from inside of the valve). E, The partial transverse aortotomy was closed incorporating the Dacron patch.

anything larger than the rectangular patch. In summary, our enlargement technique is simple and effective for avoiding patient-prosthesis mismatch and preparing patients for future valve-in-valve transcatheter AVR.

The author thanks our perfusionist, Patrick Prasch, MPS, CCP, LP, RRT, at the University of Michigan for the artwork.

\section{References}

1. Nicks R, Cartmill T, Bernstein L. Hypoplasia of the aortic root. The problem of aortic valve replacement. Thorax. 1970;25:339-46.

2. Manouguian S, Seybold-Epting W. Patch enlargement of the aortic valve ring by extending the aortic incision into the anterior mitral leaflet. New operative technique. J Thorac Cardiovasc Surg. 1979;78:402-12.

3. Imanaka K, Takamoto S, Furuse A. Mitral regurgitation late after Manouguian's anulus enlargement and aortic valve replacement. J Thorac Cardiovasc Surg. 1998;115:727-9. 